\title{
Cathepsin L promotes angiogenesis by regulating the CDP/Cux/ VEGF-D pathway in human gastric cancer
}

\author{
Tao Pan $^{1} \cdot$ Zhijian Jin ${ }^{1} \cdot$ Zhenjia Yu ${ }^{1}$ Xiongyan $\mathrm{Wu}^{1} \cdot$ Xinyu Chang ${ }^{1} \cdot$ Zhiyuan Fan $^{1} \cdot$ Fangyuan $\mathrm{Li}^{1} \cdot$ Xiaofeng Wang $^{2}$. \\ Zhen $\mathrm{Li}^{1}$ • Quan Zhou ${ }^{1}$. Jianfang $\mathrm{Li}^{1}$ • Bingya Liu ${ }^{1} \cdot$ Liping Su${ }^{1} \mathbb{C}$
}

Received: 7 February 2020 / Accepted: 24 April 2020 / Published online: 9 May 2020

(c) The Author(s) 2020

\begin{abstract}
Background Increasing evidence indicates that angiogenesis plays an important role in tumor progression. The function of cathepsin L (CTSL), an endosomal proteolytic enzyme, in promoting tumor metastasis is well recognized. The mechanisms by which CTSL has promoted the angiogenesis of gastric cancer (GC), however, remains unclear.

Methods The nuclear expression levels of CTSL were assessed in GC samples. The effects of CTSL on GC angiogenesis were determined by endothelial tube formation analysis, HUVEC migration assay, and chick embryo chorioallantoic membrane (CAM) assay. The involvement of the CDP/Cux/VEGF-D pathway was analyzed by angiogenesis antibody array, Western blot, co-immunoprecipitation (Co-IP) and dual-luciferase reporter assay.

Results In this study, we found that the nuclear CTSL expression level in GC was significantly higher than that in adjacent nontumor gastric tissues and was a potential important clinical prognostic factor. Loss- and gain-of-function assays indicated that CTSL promotes the tubular formation and migration of HUVEC cells in vitro. The CAM assay also showed that CTSL promotes angiogenesis of GC in vivo. Mechanistic analysis demonstrated that CTSL can proteolytically process CDP/Cux and produce the physiologically relevant p110 isoform, which stably binds to VEGF-D and promotes the transcription of VEGF-D, thus contributing to the angiogenesis of GC.

Conclusion The findings of the present study suggested that CTSL plays a constructive role in the regulation of angiogenesis in human GC and could be a potential therapeutic target for GC.
\end{abstract}

Keywords CTSL $\cdot$ Cux $\cdot$ VEGF-D $\cdot$ Angiogenesis

Tao Pan, Zhijian Jin and Zhenjia Yu contributed equally to this article.

Electronic supplementary material The online version of this article (https://doi.org/10.1007/s10120-020-01080-6) contains supplementary material, which is available to authorized users.

Bingya Liu

liubingya@sjtu.edu.cn

Liping Su

suliping@shsmu.edu.cn

1 Department of Surgery, Shanghai Key Laboratory of Gastric Neoplasms, Shanghai Institute of Digestive Surgery, Ruijin Hospital, Shanghai Jiao Tong University School of Medicine, Shanghai, China

2 Department of General Surgery, First People's Hospital, Shanghai General Hospital, Shanghai Jiao Tong University School of Medicine, Shanghai, China

\author{
Abbreviations \\ CTSL Cathepsin L \\ GC Gastric cancer \\ VEGF-D Vascular endothelial growth factors D \\ CDP/Cux 1 CCAAT displacement protein/cut like \\ homeobox 1 \\ FBS Fetal bovine serum
}

\section{Background}

Gastric cancer (GC) is one of the most common and deadliest types of cancer worldwide, with particularly high morbidity and mortality in China [3, 7]. Nearly, half of all patients with GC develop metastases. At present, surgery is the major curative treatment for GC; however, the prognosis of GC remains poor $[2,35]$. Therefore, the exploration of new GC treatment strategies is needed. 
Cathepsins, containing about 15 classes in human, are defined as protease enzymes with versatile functions [24]. These enzymes are vital for normal physiological processes, such as innate immunity, apoptosis, autophagy, and angiogenesis [4]. Dysregulated cathepsins can also contribute to numerous pathologies, including arthritis, pancreatitis, atherosclerosis, and cancers [6, 24]. In GC, most of cathepsins have reported to be overexpressed and are associated intimately with the proliferation and metastasis of GC cells, lymph node metastasis, and poor prognosis [13, 14, 19]. Cathepsin L (CTSL), one of the human cathepsin proteases, is primarily present within the lysosomes or secretory vesicles and predominantly exhibits endoproteolytic activity [5, 9, 31]. Our study, however, showed that CTSL also is found in the nucleus of GC; overexpression of nuclear CTSL in tumor tissues was significantly associated with differentiation, local invasion, TNM stage, lymph metastasis, and shorter survival of GC patients. Moreover, loss- and gainof-function assays demonstrated that CTSL promotes tumor angiogenesis in GC.

Accumulating evidence has revealed that tumor progression is strongly associated with angiogenesis, which plays a critical role in tumor growth, relapse, and metastasis. Vascular endothelial growth factor (VEGF) is the key regulator of angiogenesis, and tumor-derived VEGFs play a central role in tumor angiogenesis [16, 27]. VEGF-D, a member of the VEGF family, is identified as a lymph-angiogenic growth factor and participates in the growth of lymphatic vessels as well as in angiogenesis [1,28]. VEGF-D binds to the tyrosine kinase receptors (TKRs) VEGFR-2 and VEGFR-3 that are expressed specifically on the surface of GC cells, which then activates the downstream signaling pathways, including the phosphatidylinositol 3 kinase (PI3K)/AKT, protein kinase $\mathrm{C}(\mathrm{PKC})$, and mitogen-activated protein kinase (MAPK) pathways [21], to induce angiogenesis within the solid tumors, promoting tumor growth and distant metastasis [17, 34]. In our study, we found that CTSL could proteolytically cleave the CDP/Cux p200 and generate $\mathrm{CDP} / \mathrm{Cux} \mathrm{p} 110$, which contributed to the transcriptional activation of VEGF-D and promoted tumor angiogenesis in GC. These results indicated the novel mechanism of CTSL in the progression of GC and revealed that it could act as a new therapeutic target in cancer therapy.

\section{Methods}

\section{Cell culture and patient samples}

The GC cell lines AGS and NCI-N87 used in our study were purchased from the American Type Culture Collection (ATCC) and SUN-1, MGC803, MKN45, MKN28, HGC27, and GES-1 were purchased from Shanghai Institutes for
Biological Sciences, Chinese Academy of Sciences. All GC cell lines were authenticated by short-tandem repeat analysis and had negative results for mycoplasma. SUN-1, AGS, NCI-N87, MKN45, MKN28, and HGC27 were cultured at $37{ }^{\circ} \mathrm{C}$ in a humidified atmosphere of $5 \% \mathrm{CO}_{2}$ with RPMI1640 medium containing $10 \%$ fetal bovine serum with 100 $\mathrm{U} / \mathrm{ml}$ penicillin and $100 \mathrm{U} / \mathrm{ml}$ streptomycin. GES-1 and MGC803 were cultured at $37{ }^{\circ} \mathrm{C}$ in a humidified atmosphere of $5 \% \mathrm{CO}_{2}$ with Dulbecco's Modified Eagle Medium (DMEM) containing 10\% fetal bovine serum with $100-\mathrm{U} / \mathrm{ml}$ penicillin and 100-U/ml streptomycin.

A total of 174 patients in this study underwent resection of the GC at Shanghai Ruijin Hospital. All samples were obtained with the patients' informed consent, and the samples were histologically confirmed.

\section{RNA extraction and qRT-PCR}

Total RNA was extracted with Trizol reagent (Invitrogen, Carlsbad, CA, USA), which was reversely transcribed into cDNA using a Reverse Transcription system (TOYOBO, Kita-ku, Osaka, Japan). Quantitative real-time polymerase chain reaction (qRT-PCR) was performed to quantify CTSL and VEGF-D mRNA levels with the SYBER Green PCR Master Mix (Applied Biosystems, Foster City, CA, USA). Glyceraldehyde 3-phosphate dehydrogenase (GAPDH) were used as the endogenous reference and used the $2^{-\left(\Delta \Delta C_{t}\right)}$ method to calculate the relative abundance of RNA compared with GAPDH expression.

\section{Western blot}

Protein was extracted from cells using radioimmunoprecipitation (RIPA) buffer (Thermo, Rockford, IL, USA) and cell debris were removed by centrifugation. The extracts were quantified using Pierce BCA Protein Assay Kit (Thermo) and then boiled the extracts in sodium dodecyl sulfate (SDS) gel-loading buffer containing $10 \% \beta$-mercaptoethanol. The proteins were separated in 10\% SDS-polyacrylamide gel electrophoresis (SDS-PAGE) gels and transferred them onto immobilon polyvinylidene fluoride (PVDF) membranes (Millipore, Burlington, MA, USA), which were probed with rabbit mAbs against mouse GAPDH (1:5000; 60004-1-Ig; ProteinTech Group, Chicago, IL, USA), CDP/Cux1 (1:1000; sc514008X; Santa Cruz Biotechnology, Dallas, TX, USA), VEGF-D (1:1000; 26915-1-AP; ProteinTech Group, Chicago, IL, USA), and CTSL (1:1000, ab58991; Abcam, Cambridge, MA, USA) and then incubated with horseradish peroxidase (HRP)-conjugated goat anti-rabbit (SA00001-2) and anti-rabbit immunoglobulin $\mathrm{G}(\mathrm{IgG}, \mathrm{SA00001-1)}$ antibody (1:10,000; 13099-1-AP; ProteinTech Group, Chicago, IL, USA), which was followed by Pierce ${ }^{\mathrm{TM}}$ ECL Western Blotting Substrate (Thermo). 


\section{Plasmids construction and transfection}

Two CTSL small hairpin RNAs (shRNA, sh-CTSL \#1 and sh-CTSL \#2) and negative control (Ctrl) sequences were designed and constructed into a hU6-MCS-Ubiquitin-EGFP-IRES-puromycin plasmid. HGC27, MGC803, MKN28, and MKN45 cells were transfected with shRNA plasmids using Lipofectamine 3000 reagent (Invitrogen) according to the manufacturer's protocol. CTSL cDNA was subcloned into the CN550-pLOV-EF1a-PuroRCMV-eGFP-2A-3FLAG plasmid (Obio Technology Co. Ltd., Shanghai, China). CTSL plasmid and corresponding empty vector were transfected the into GC cells (HGC27, MGC803, AGS, and SUN-1) using Lipofectamine 3000 reagent (Invitrogen) following the manufacturer's protocol. The stably transfected cell lines were selected by puromycin.

\section{Immunohistochemistry staining}

IHC staining was performed on the tissue microarray according to a previously reported standard protocol used in Shanghai Institute of Digestive Surgery [36]. In brief, freshly cut 5 - $\mu \mathrm{m}$-thick tissue microarray was stained with antibodies against CTSL (1:200, ab203028, Abcam, Cambridge, MA, USA), VEGF-D (1:1000; 26915-1-AP; ProteinTech Group, Chicago, IL, USA) and PECAM1 (1:200, ab268100; Abcam, Cambridge, MA, USA). Two independent boardcertified pathologists evaluated the protein expression levels in an unbiased fashion. The staining intensity and percentage were used to score the overall tissue sections and the intensity was graded as follows: 0 points (no staining), 1 point (light-brown staining), 2 points (brown staining), and 3 points (dark-brown staining). We divided the percentage of positive cell number into four grades: 1 points $(<5 \%), 2$ point (5-30\%), 3 points (31-60\%), and 4 points (61-100\%). We calculated the staining score as follows: overall staining score $=$ intensity score $\times$ percentage score. A final score $\leq 3$ was considered to be negative staining, and a score $>3$ was considered to be positive staining.

\section{Transient transfection and dual-luciferase reporter assay}

HGC27-Vector and HGC27-p110Cux1 cells were seeded in 24-well plates and transfected the cells with pGL3-VEGF-D, pGL3-VEGF-D-del 1, pGL3-VEGF-D-del 2, pGL3-VEGFD-del 3, and pRL-TK plasmid using a Lipofectamine ${ }^{\mathrm{TM}} 3000$ Transfection Reagent (Invitrogen) according to the manufacturer's instructions. Three days after transfection, dual-luciferase reporter assay was performed using Dual-Luciferase ${ }^{\circledR}$
Reporter Assay System (Promega, Madison, WI, USA) according to the manufacturer's instructions.

\section{Endothelial tube formation analysis}

Human umbilical vein endothelial cells (HUVECs) were cultured in tumor supernatants from each cell line in 96-well plates at a density of $1 \times 10^{4}$ cells per well for $6 \mathrm{~h}$. Plates were pre-coated with 50- $\mu \mathrm{L}$ Matrigel (R\&D Systems, 3533010-02) at $37{ }^{\circ} \mathrm{C}$ for $1 \mathrm{~h}$. After a 6-h incubation, tubule images were acquired and analyzed by Image-Pro Plus software, which we quantified by counting the number of cell junctions and tubes in 10 randomly chosen fields of view. Data were obtained from three independent experiments.

\section{HUVEC migration assay}

HUVECs were suspended in serum-free medium $\left(1 \times 10^{5}\right.$ cells/insert) and added to the upper chamber of the 24-well insert (membrane pore size, $8 \mu \mathrm{m}$; Corning Life Science, Tewksbury, MA, USA). Medium containing $10 \%$ serum was added to the lower chamber. After incubation for $12 \mathrm{~h}$, the cells that had migrated to the bottom of the membranes were fixed and stained them with $0.1 \%$ crystal violet for $30 \mathrm{~min}$.

\section{Chick embryo chorioallantoic membrane assay}

A chorioallantoic membrane (CAM) was performed as previously described [8]. After cutting a round window into an egg, $30 \mu \mathrm{L}$ of cell culture supernatant was dropped onto a filter paper disk and sealed with transparent tape for 3 days. On day 10, the eggs were photographed with a MacroPATH dissecting microscope (Milestone, Sorisole, Italy) and the number of blood vessels around the filter paper disc was counted.

\section{Angiogenesis antibody array}

A Human Angiogenesis Antibody Array C1000 (RayBiotech, Norcross, GA, USA) was used to analyze the conditioned medium (CM), following the manufacturer's protocol. The results of the antibody array were quantified with a chemiluminescence system (Bio-Rad, Hercules, CA, USA) similar to the Western blotting procedure.

\section{In vivo tumorigenesis}

Four-week-old male BALB/c nude mice were purchased from the Institute of Zoology Chinese Academy of Sciences and housed at a specific pathogen-free environment in the Animal Laboratory Unit, Ruijin Hospital, China. HGC27 cells transduced by sh-CTSL or sh-Ctrl $\left(2 \times 10^{7}\right)$ were harvested and resuspended in $500 \mu \mathrm{L}$ of PBS. Cells 
$\left(4 \times 10^{6} / 100 \mu \mathrm{L}\right.$ PBS $)$ were subcutaneously or peritoneally injected into the mice. For tumorigenesis in vivo, all mice were killed after 28 days, and subcutaneous lumps were removed, imaged, and placed in $4 \%$ formaldehyde for paraffin embedding. For peritoneal dissemination model, all mice were killed after 30 days and peritoneal metastasis nodules were counted.

\section{Statistical analysis}

All experiments were repeated at least three times, and the results were summarized as means $\pm \mathrm{SD}$. Student $t$ test and one-way analysis of variance (ANOVA) were used to analyze the data and Chi-square tests were used to analyze categorical variables. All statistical tests were performed with SPSS 25.0 (SPSS, Chicago, IL, USA) and $p$ values $<0.05$ were regarded as indicating statistically significant differences $(* p<0.05, * * p<0.01, * * * p<0.001)$.

\section{Results}

\section{Overexpressed nuclear CTSL is positively associated with vessel density in human GC tissues and predicts poor prognosis of GC patients}

Previous studies have demonstrated that CTSL is involved in a wide range of human malignancies, including ovarian, breast, prostate, lung, pancreatic, and colon cancers [30]. In accordance with previous studies, bioinformatic analysis of CTSL expression profiles through Gene Expression Profiling Interactive Analyses (GEPIA; https://gepia.cancer-pku.cn) showed that the CTSL mRNA expression levels were significantly increased in majority of tumor types (Supplementary Fig. 1). Notably, CTSL was obviously upregulated in GC tissues (Fig. 1a). Moreover, bioinformatic analyses showed that expression of CTSL was positively related to TNM stage (Fig. 1b). We then examined CTSL protein expression in 134 pairs of GC tissues (GC cohort 1) and examined corresponding nontumor tissues by IHC. Typical immunostaining of CTSL in GC tissues and corresponding non-tumor tissues is shown in Fig. 1c. We primarily identified positive staining of CTSL protein in the cytoplasm and nuclei of GC cells. CTSL has been demonstrated to promote the development of GC [20, 22]; however, the exact role of nuclear CTSL in GC progression remains undefined. To further evaluate the function of nuclear CTSL in GC, we next analyzed the expression level of the nuclear fractions of CTSL in GC, and the results showed that the average expression score for the nuclear fractions of CTSL was significantly higher in GC tissues than that in adjacent nontumor tissues (Fig. 1d). Furthermore, we analyzed the correlation of nuclear CTSL with the clinical parameters of GC, as shown in Table 1. A high nuclear CTSL staining score was associated with more poor differentiation, local invasion, positive lymphatic metastasis status, and higher TNM stage. In addition, we assessed the correlation between CTSL expression and the survival of GC patients. Kaplan-Meier Plotter analysis (https://kmplot.com/ analysis) showed that CTSL expression was inversely proportional to survival (Fig. 1e, f). We further identified that prognosis of cases with high nuclear CTSL expression was worse (Fig. 1g) and multivariate analyses of different prognostic parameters revealed that nuclear CTSL expression was an independent factor for overall survival $(p=0.026)$ (Fig. 1h). It has been reported that cysteine cathepsins play an important role in angiogenesis [25]. To further define the clinic role of CTSL in angiogenesis of GC, we detected the VD of GC tissues by IHC staining of PECAM1 (also called CD31, a marker of angiogenesis). The result showed that VD in tumor tissue was significantly higher than in nontumor tissues (Fig. 1i, j), and further analysis showed that the nuclear CTSL expression was positively related to the VD of GC tissues (Fig. 1k). Similarly, bioinformatic analyses of the correlation between CTSL and PECAM1 through GEPIA (Fig. 11) confirmed these findings, which indicated that nuclear CTSL may play a vital role in the angiogenesis of GC. Overall, these data suggest that upregulation of nuclear CTSL is positively correlated with VD in human GC and predicts poor prognosis of GC patients.

\section{CTSL promotes the tubular formation and migration of HUVEC cells}

Given a potential link between CTSL overexpression and angiogenesis in GC, we determined whether knockdown or ectopic overexpression of CTSL in GC cells could affect the tubular formation and migration of endothelial cells. We identified the expression levels of CTSL in seven GC cell lines and one human gastric epithelial cell line (GES-1). Most GC cell lines expressed relatively high levels of CTSL (Supplementary Fig. 2A and Supplementary Fig. 2B). To determine whether the expression level of CTSL changed in GC cells and contributed to endothelial cells tubule formation and migration, we established an in vitro co-culture system (Supplementary Fig. 2C), in which HUVECs were indirectly cocultured with GC cells and separated from GC cells by a semipermeable membrane (pore size of $0.6 \mu \mathrm{m}$ ). Interestingly, we found that CTSL-knockdown GC cells elicited significantly less tubule formation by HUVECs compared with their respective control group (Fig. 2a, b); whereas, CTSL-overexpressing GC cells stimulated more tubule formation by HUVECs in comparison with their respective control group (Fig. 2c, d). Tumor cells may regulate HUVEC tubule formation by altering endothelial cell migration. We then performed Transwell assays, and the result revealed that overexpression of CTSL in GC cells promoted 
Fig. 1 Overexpressed nuclear CTSL was positively associated with VD in human GC tissues and predicts poor prognosis of GC patients. a Box plots of 408 GC samples (red) and 211 normal samples (gray) revealed upregulation of CTSL in GC compared with normal tissue. b Violin plots revealed that CTSL expression was significantly associated TNM stages. c Representative IHC staining with CTSL antibody in GC and paired adjacent nontumor tissues. Magnification: $\times 200$ and $\times 400$. d Analysis of CTSL expression in 134 pairs of GC patients (GC cohort 1) showed that CTSL expression was significantly high in GC tissues compared with adjacent nontumor tissues. $\mathrm{e}$ The overall survival and $\mathbf{f}$ disease-free survival analyses were plotted using Kaplan-Meier Plotter for patients with GC. $\mathbf{g}$ Kaplan-Meier analysis of the overall survival of 134 patients with GC in GC cohort 1. The log-rank test revealed statistical significance between the low nuclear CTSL expression group $(n=46)$ and the high nuclear CTSL expression group $(n=88)$. h Multivariate analysis of different prognostic parameters for GC patients (GC cohort 1). i Representative IHC staining with PECAM1 antibody in GC and paired adjacent nontumor tissues. Magnification: $\times 200$ and $\times 400$. j Analysis of VD in 134 pairs of GC patients (GC cohort 1) showed that VD was significantly high in GC tissues compared with adjacent nontumor tissues. $\mathbf{k}$ Correlation analysis of CTSL and VD showed that CTSL was positively correlated with VD in GC. I Validation of the positive correlation between CTSL and VD in GEPIA

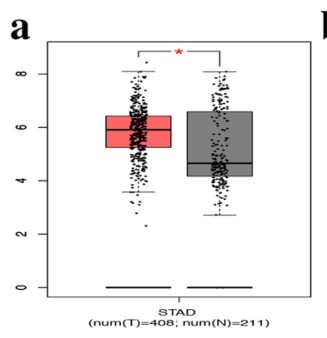

c

E
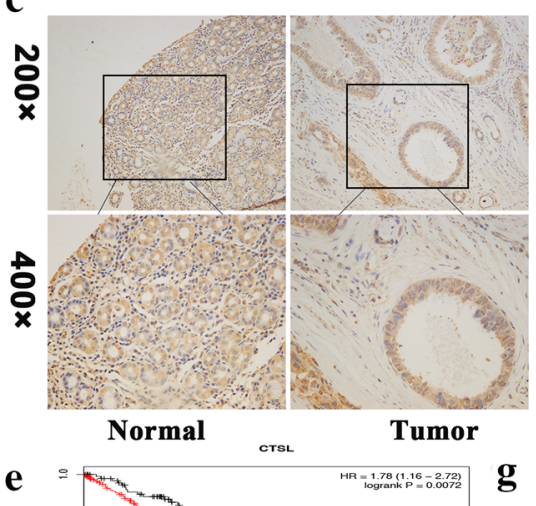

g

f

f
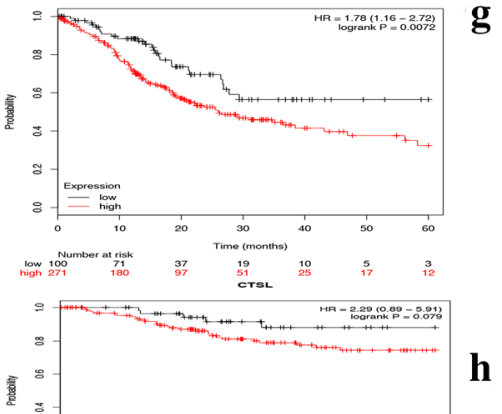

d
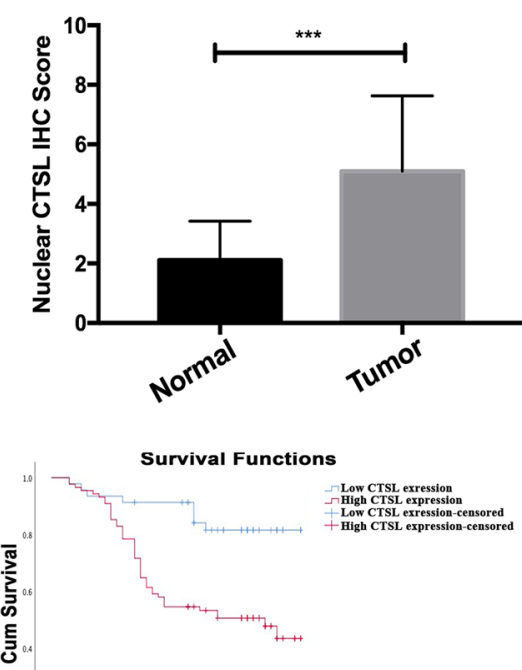

$\mathbf{n}$

$n=134$

$p<0.001$
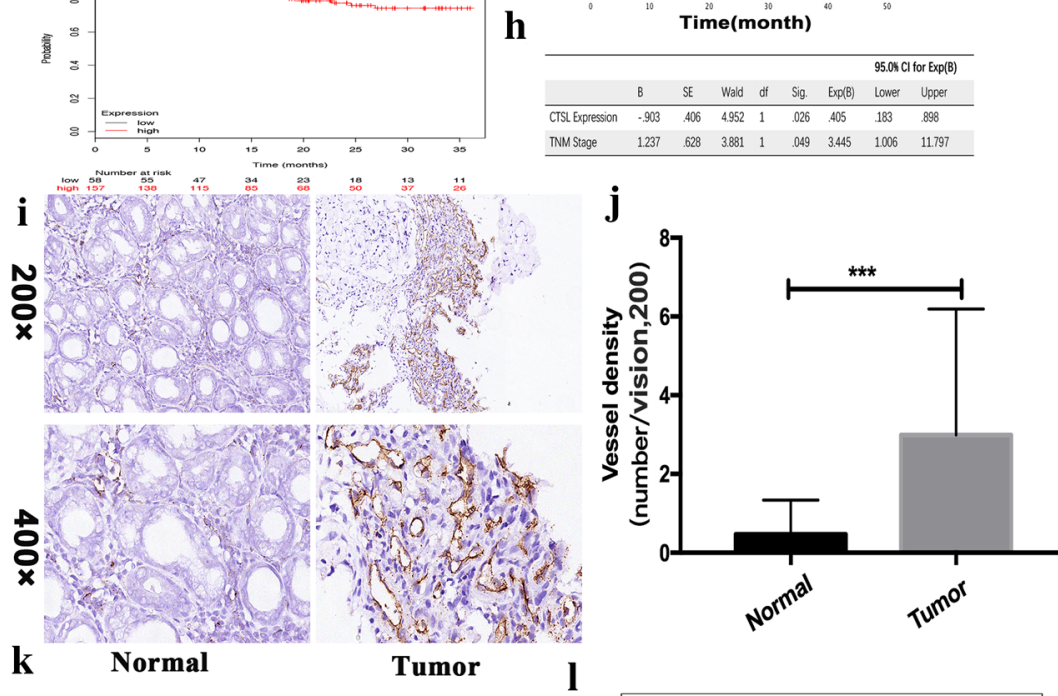

j

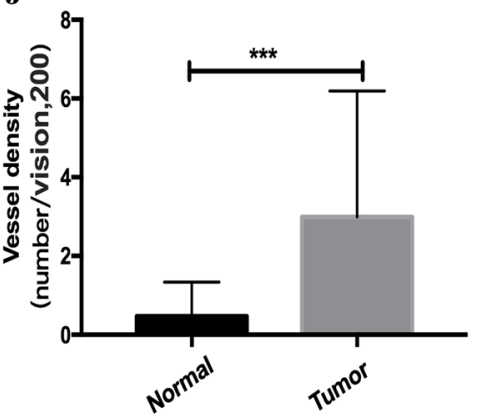

k Normal

$r=0.3632$

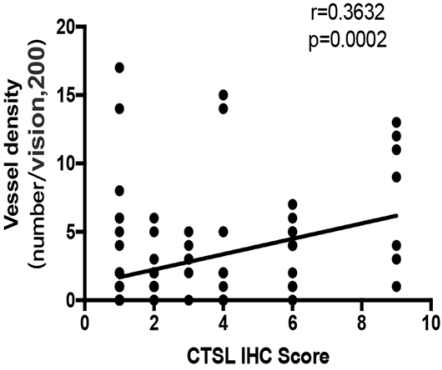


Table 1 Correlation between the clinicopathological features and expression of CTSL

\begin{tabular}{|c|c|c|c|c|}
\hline \multirow[t]{2}{*}{ Clinicopathologic parameters } & \multirow{2}{*}{$\begin{array}{l}\text { Number of } \\
\text { cases } \\
134\end{array}$} & \multicolumn{2}{|c|}{ Nuclear CTSL immunostaining } & \multirow[t]{2}{*}{$p$} \\
\hline & & $\begin{array}{l}\text { Weak positive } \\
(n=46)\end{array}$ & $\begin{array}{l}\text { Strong positive } \\
(n=88)\end{array}$ & \\
\hline \multicolumn{5}{|l|}{ Gender } \\
\hline Male & 95 & 32 & 63 & \multirow[t]{2}{*}{0.806} \\
\hline Female & 39 & 14 & 25 & \\
\hline \multicolumn{5}{|l|}{ Age (years) } \\
\hline$\geq 60$ & 75 & 30 & 45 & \multirow[t]{2}{*}{0.119} \\
\hline$<60$ & 59 & 16 & 43 & \\
\hline \multicolumn{5}{|l|}{ Tumor size $(\mathrm{cm})$} \\
\hline$\geq 5$ & 95 & 32 & 63 & \multirow[t]{2}{*}{0.806} \\
\hline$<5$ & 39 & 14 & 25 & \\
\hline \multicolumn{5}{|l|}{ Lauren classification } \\
\hline Intestinal & 78 & 28 & 50 & \multirow[t]{2}{*}{0.652} \\
\hline Diffuse & 46 & 18 & 38 & \\
\hline \multicolumn{5}{|l|}{ Differentiation } \\
\hline Poorly undifferentiated & 23 & 17 & 6 & \multirow[t]{2}{*}{$<0.001$} \\
\hline Well moderately & 111 & 29 & 82 & \\
\hline \multicolumn{5}{|l|}{ Local invasion } \\
\hline $\mathrm{T} 1, \mathrm{~T} 2$ & 20 & 16 & 4 & \multirow[t]{2}{*}{$<0.001$} \\
\hline $\mathrm{T} 3, \mathrm{~T} 4$ & 114 & 30 & 84 & \\
\hline \multicolumn{5}{|l|}{ Lymph metastasis } \\
\hline No & 26 & 15 & 11 & \multirow[t]{2}{*}{0.005} \\
\hline Yes & 108 & 31 & 77 & \\
\hline \multicolumn{5}{|l|}{ TNM stage } \\
\hline I, II & 27 & 22 & 5 & \multirow[t]{2}{*}{$<0.001$} \\
\hline III, IV & 107 & 24 & 83 & \\
\hline
\end{tabular}

the migration of HUVECs (Fig. 2e, f); whereas, knockdown of CTSL expression in GC cells led to the reduced migratory capacity of HUVECs (Fig. 2g, h). Furthermore, a woundhealing assay confirmed that knockdown of CTSL in GC cells markedly increased HUVECs migration (Fig. 2i-m, Supplementary Fig. 3A); whereas, overexpression had the opposite effect (Supplementary Fig. 3B and Fig. 2n). These results indicate that CTSL could promote the tubular formation and migration of HUVEC cells in vitro.

\section{CTSL promotes angiogenesis and tumor growth in vivo}

To determine the effect of CTSL on angiogenesis in vivo, we performed a chick embryo CAM assay, which has emerged as a standard in in vivo angiogenesis assay [32]. Treatment with CM from the CTSL-knockdown GC cells significantly reduced the vessel number compared with their respective control group (Fig. 3a, b); whereas, CM from CTSL-overexpressing GC cells induced the formation of more vessels than that from their respective control group (Fig. 3c, d). In addition, we further identified the tumor-promoting effect of
CTSL in vivo. We subcutaneously transplanted HGC27/shCTSL and HGC27/sh-Ctrl cells into nude mice. As shown in Fig. 3e-h, the tumors generated by HGC27/sh-CTSL cells exhibited smaller volume and less weight than those generated by HGC27/sh-Ctrl cells. Moreover, we further detected VD in subcutaneous tumors derived from HGC27/sh-CTSL and HGC27/sh-Ctrl cells. Anti-CD31 immunostaining revealed that vascellum in tumors from HGC27/sh-Ctrl cells was more developed; however, less vascellum formed in tumors from HGC27/sh-CTSL cells (Fig. 3i). Consistently, the assessment of averaged VD per field in each group indicated a threefold higher level in tumors from HGC27/ sh-Ctrl cells when compared with tumors from HGC27/shCTSL cells (Fig. 3j). Thus, these findings suggest that CTSL in GC cells enhances tumor progression by promoting the angiogenesis of GC.

\section{CTSL promotes angiogenesis by transcriptionally activating of VEGF-D via p110 Cux1 in GC cells}

The angiogenesis of a tumor is regulated by the coordination of the angiogenic and anti-angiogenic factors secreted by 
Fig. 2 CTSL promoted the tubular formation and migration of HUVEC cells. a, b In vitro tubular formation to investigate the effect of CM from GC cells after transfection with CTSL shRNA plasmids. (MGC803, HGC27, MKN28, and MKN45) on HUVEC tubule formation. Data are presented as the mean $\pm \mathrm{SD} ; n=3$ independent experiments. c, $\mathbf{d}$ In vitro tubular formation to investigate the effect of CM from GC cells after transfection with CTSLexpressing plasmids (MGC803, HGC27, AGS, and SUN-1) on

HUVEC tubule formation. Data are presented as the mean $\pm \mathrm{SD}$; $n=3$ independent experiments. e, f In vitro Transwell migration assay to investigate the effect of CM from GC cells after transfection with CTSL shRNA plasmids (MGC803, HGC27, MKN28, and MKN45) on the metastatic abilities of HUVEC. Data are presented as the mean $\pm \mathrm{SD} ; n=3$ independent experiments. $\mathbf{g}, \mathbf{h}$ In vitro Transwell migration assay to investigate the effect of $\mathrm{CM}$ from GC cells after transfection with CTSL-expressing plasmids (MGC803, HGC27, AGS, and SUN-1) on the metastatic abilities of HUVEC. Data are presented as the mean $\pm \mathrm{SD}$; $n=3$ independent experiments. i-m Scratch wound-healing motility assays were performed to observe the changes in the migration of HUVEC after stimulation with $\mathrm{CM}$ from GC cells after transfection with CTSL shRNA plasmids (MGC803, HGC27, and MKN28) Data are presented as the mean $\pm \mathrm{SD} ; n=3$ independent experiments. $\mathbf{n}$ Scratch wound-healing motility assays were performed to observe the changes in the migration of HUVEC after stimulated with CM from GC cells after transfection with CTSL-expressing plasmids (AGS). Data are presented as the mean $\pm \mathrm{SD} ; n=3$ independent experiments $\mathbf{a}$

ป̛̃

\section{b}
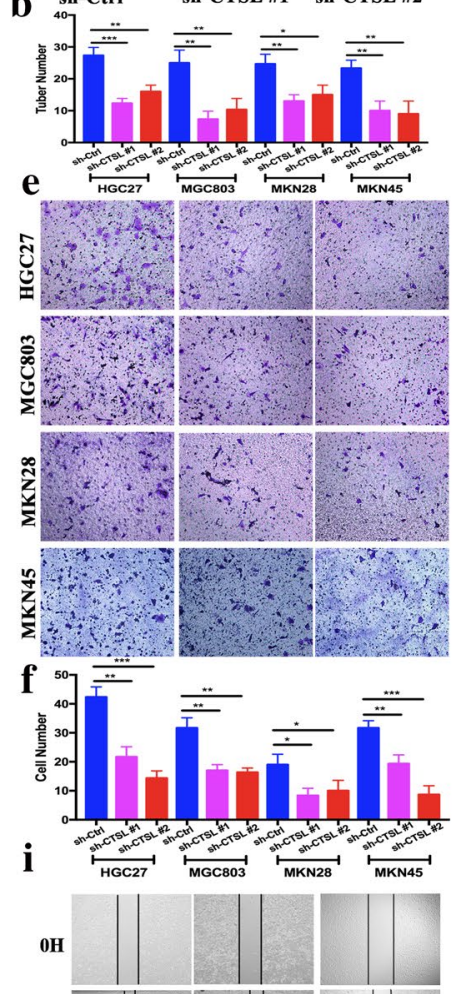

స్్ㅍ

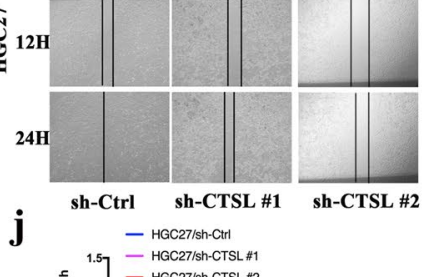

m

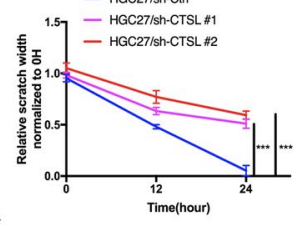

- MKN28/sh-CrrI

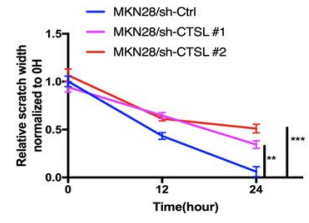

c

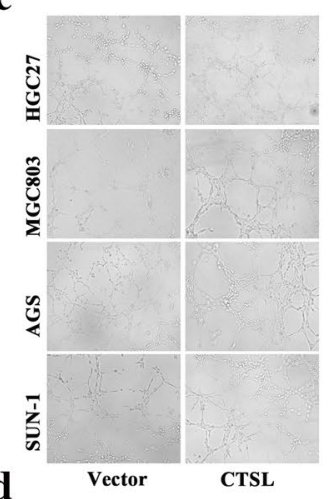

d

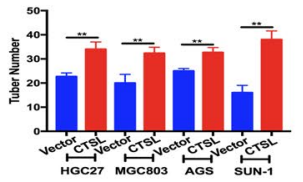

g

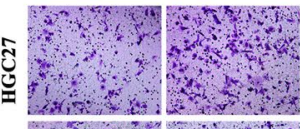

:

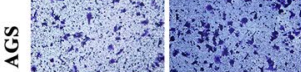

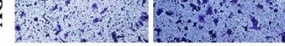
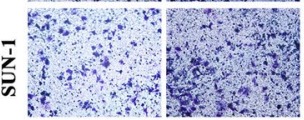

h
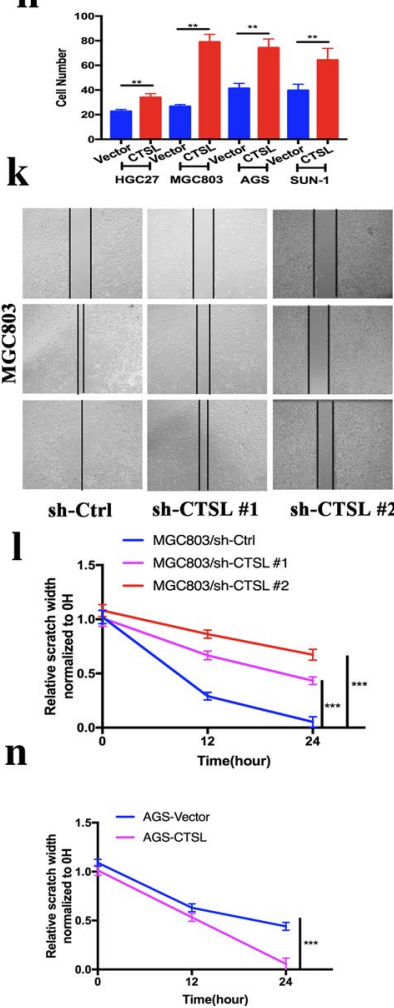


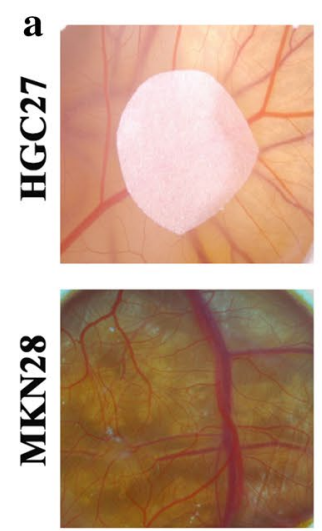

sh-Ctrl
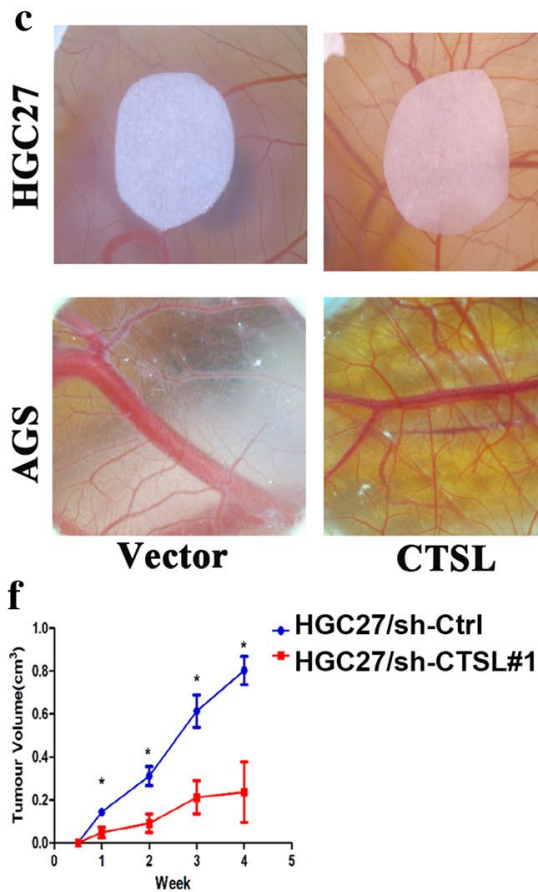

h

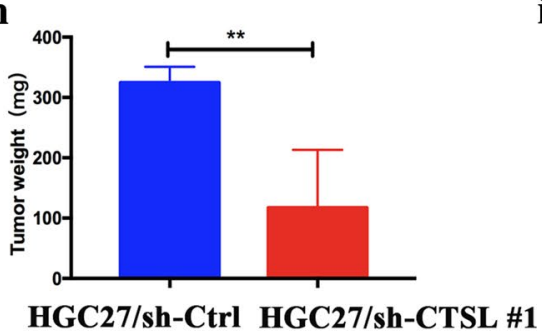

j

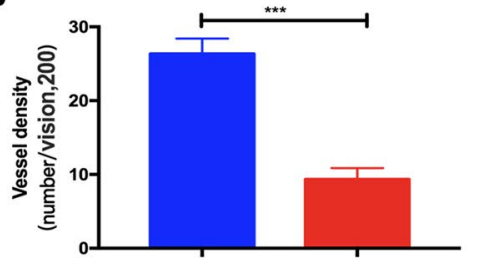

HGC27/sh-Ctrl HGC27/sh-CTSL \#1 b

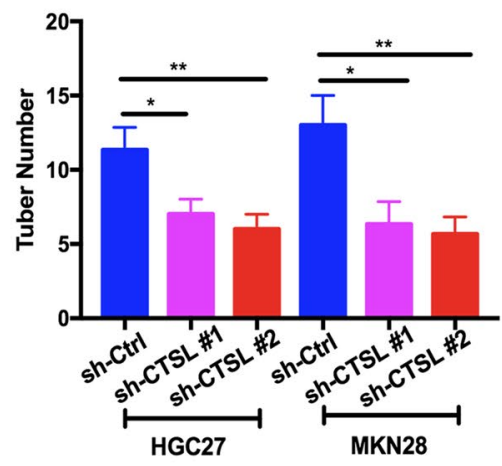

sh-CTSL \#2

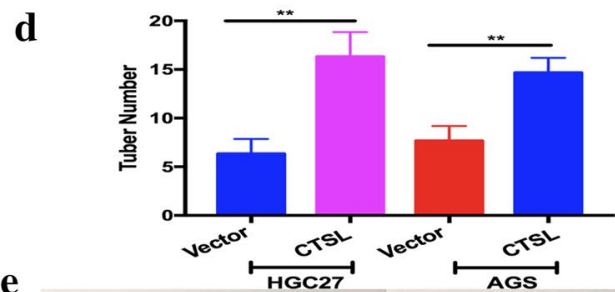

e

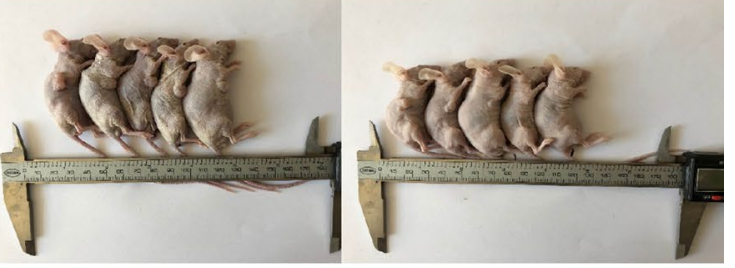

HGC27/sh-Ctrl HGC27/sh-CTSL \#1

g

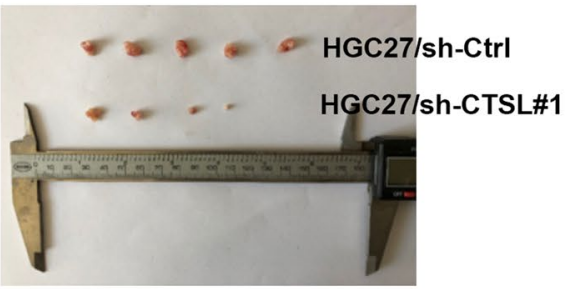

\section{HGC27/sh-Ctrl}

HGC27/sh-CTSL \#1
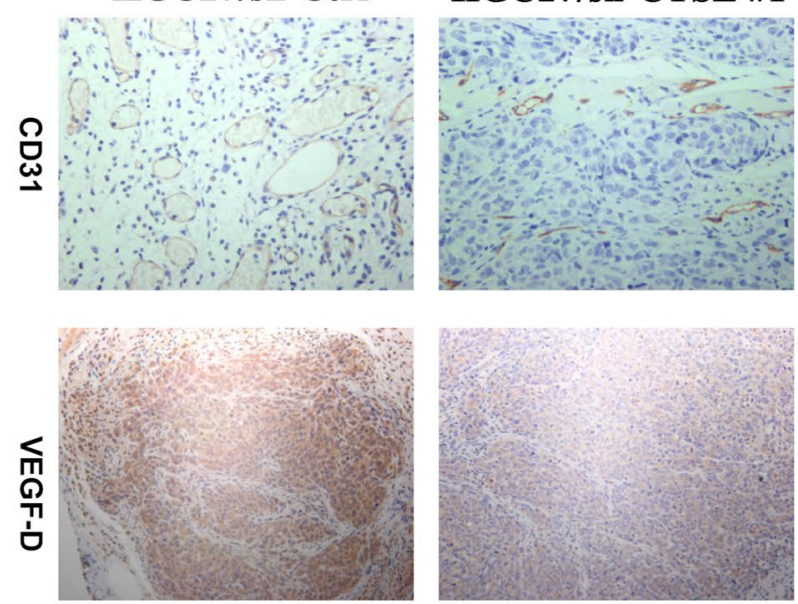
4Fig. 3 CTSL promoted angiogenesis and tumor growth in vivo. a, b The CAM assay was performed to investigate the effect of CM from GC cells after transfection with CTSL shRNA plasmids (HGC27 and MKN28) on HUVEC tubule formation in vivo. $\mathbf{c}, \mathbf{d}$ The CAM assay was performed to investigate the effect of $\mathrm{CM}$ from $\mathrm{GC}$ cells after transfection with CTSL-expressing plasmids (HGC27 and AGS) on HUVEC tubule formation in vivo. e, $\mathbf{f}$ Mice were injected with HGC27 cells transfected with CTSL shRNA plasmids (sh-CTSL \#1) or sh-Ctrl, and tumor volumes were monitored weekly. $\mathbf{g}, \mathbf{h}$ Average tumor weights from nude mice injected with HGC27/sh-CTSL cells or HGC27/sh-Ctrl cells. i Representative images of CD31 (PECAM1) and VEGF-D immunostaining of tumor samples from different tumor model mice groups. $\mathbf{j}$ Analysis of VD in different tumor model mice groups showed that VD was significantly higher in tumors from HGC27/sh-Ctrl compared with tumors from HGC27/sh-CTSL

tumor cells or stromal cells in tumor microenvironment. To identify the factors from GC cells involved in the angiogenesis of tumors, we performed a human angiogenesis antibody array on CM from HGC27/sh-CTSL and HGC27/sh-Ctrl cells. The result showed a remarkable decrease in VEGF-D in CM from HGC27/sh-CTSL cells (Supplementary Fig. 4A and 4B). We, therefore, quantified the expression level of VEGF-D in HGC27/sh-CTSL cells and HGC27/sh-Ctrl cells by qRT-PCR, and we found a fivefold reduction of VEGF-D mRNA in HGC27/sh-CTSL cells compared with HGC27/sh-Ctrl cells (Fig. 4a). In addition, we collected the CM from HGC27/sh-CTSL cells and HGC27/sh-Ctrl cells and detected the protein expression of VEGF-D in CM by enzyme-linked immunosorbent assay (ELISA). The result also showed that silencing CTSL led to a significant decrease in VEGF-D secretion by GC cells (Fig. 4b).

CCAAT-displacement protein/cut homeobox (CDP/ $\mathrm{Cux}$ ) belongs to a family of transcription factors and has four evolutionarily conserved DNA binding domains: the Cut homeodomain (HD) and the three Cut repeats (CR1, CR2, and CR3) [11]. The full-length protein, named p200 Cux1, is abundant but only transiently binds DNA [23]. P110 Cux1, proteolytic processing of p200 Cux1 by CTSL, stably interacts with DNA, and functions as a transcriptional repressor or activator depending on promoter context [26, 29]. To identify whether upregulating CTSL expression in GC cells could increase CDP/Cux degradation to p110 Cux1, we performed Western blot to detect a change in the protein level of CDP/Cux. We found that overexpression of CTSL in GC cells (MGC803 and HGC27) promoted the cleavage of CDP/Cux and significantly increased the expression of p110 Cux 1 isoform (Supplementary Fig. 4C and 4D). To further determine whether p110 Cux 1 could directly bind the VEGF-D promoter and transcriptionally activate its expression in GC cells, we next analyzed the promoter region of VEGF-D, which ranged from -2000 to $+100 \mathrm{bp}$ with respect to the transcription start site (TSS), using the JASPAR database (Fig. 4c). We identified three putative p110 Cux1 binding sites (score $>5)$ at site $\mathrm{A}(-1799$ to -1790$)$, site B ( -903 to -894$)$, and site C ( -406 to -397$)$. Dual-luciferase reporter assay showed that p110 Cux 1 significantly increased the relative luciferase activity compared with the control group by cotransfecting p110 Cux 1 and the fulllength promoter of VEGF-D, the promoter 1 vector (deletion at site B: $-903 \mathrm{bp}$ to $-894 \mathrm{bp}$ ), or the promoter 2\# vector (deletion site $\mathrm{C}:-406$ to -397 ). Deletion at site $\mathrm{A}$ ( -1799 bp to $-1790 \mathrm{bp}$ ), however, significantly decreased p110 Cux1-induced VEGF-D promoter activity (Fig. 4d), which demonstrated that $110 \mathrm{Cux} 1$ could bind to the promoter of VEGF-D at -1799 to $-1790 \mathrm{bp}$ and then could activate VEGF-D transcription.

To further evaluate whether CTSL promotes the angiogenic ability of HUVECs through the CDP/Cux/VEGF-D pathway, we first established an in vitro co-culture system. We indirectly co-cultured HUVECs with GC cells (HGC27 and MGC803) after transfection with sh-CTSL, p110 Cux1-expressing vector, or Si-VEGF-D. Next, we performed tubule formation and Transwell assays, and the results revealed that upregulation of p110 Cux 1 in GC cells promoted the tubule formation and migratory ability of HUVECs, which could be reversed by co-transfection with Si-VEGF-D (Fig. 4e-h). To ascertain whether CTSL promotes the transcription of VEGF-D by increasing the expression of p110 Cux 1, we next performed Western blot and the results showed that the overexpression of $\mathrm{p} 110$ Cux 1 can rescue the sh-CTSL-mediated suppression of VEGF-D in HGC27 and MGC803 GC cells (Fig. 4i-1). In general, we found that CTSL enhances the cleavage of $\mathrm{CDP} / \mathrm{Cux}$ to $\mathrm{p} 110 \mathrm{Cux} 1$, which promotes the transcriptional activation of VEGF-D to subsequently induce angiogenesis of GC.

\section{CTSL expression is positively correlated with VEGF-D in GC tissues}

We further examined the correlation between the expression level of CTSL and VEGF-D in human GC. We performed IHC analysis to check VEGF-D expression in 157 GC patients (GC cohort 2), and the results revealed that VEGF-D expression was significantly upregulated in GC tissues compared with adjacent non-tumor tissues (Fig. 5a, b). Moreover, we found that the expression level of VEGF-D in GC was positively correlated with VD and the expression of nuclear CTSL (Fig. 5c, d). Similarly, bioinformatic analysis of the correlation of VEGF-D and VD revealed that VEGF-D was positively correlated with VD in GC (Fig. 5e). In addition, we analyzed the correlation between CTSL and VEGF-D in GC using TCGA data. As shown in Fig. 5f, CTSL expression was positively correlated with the expression of VEGF-D. 


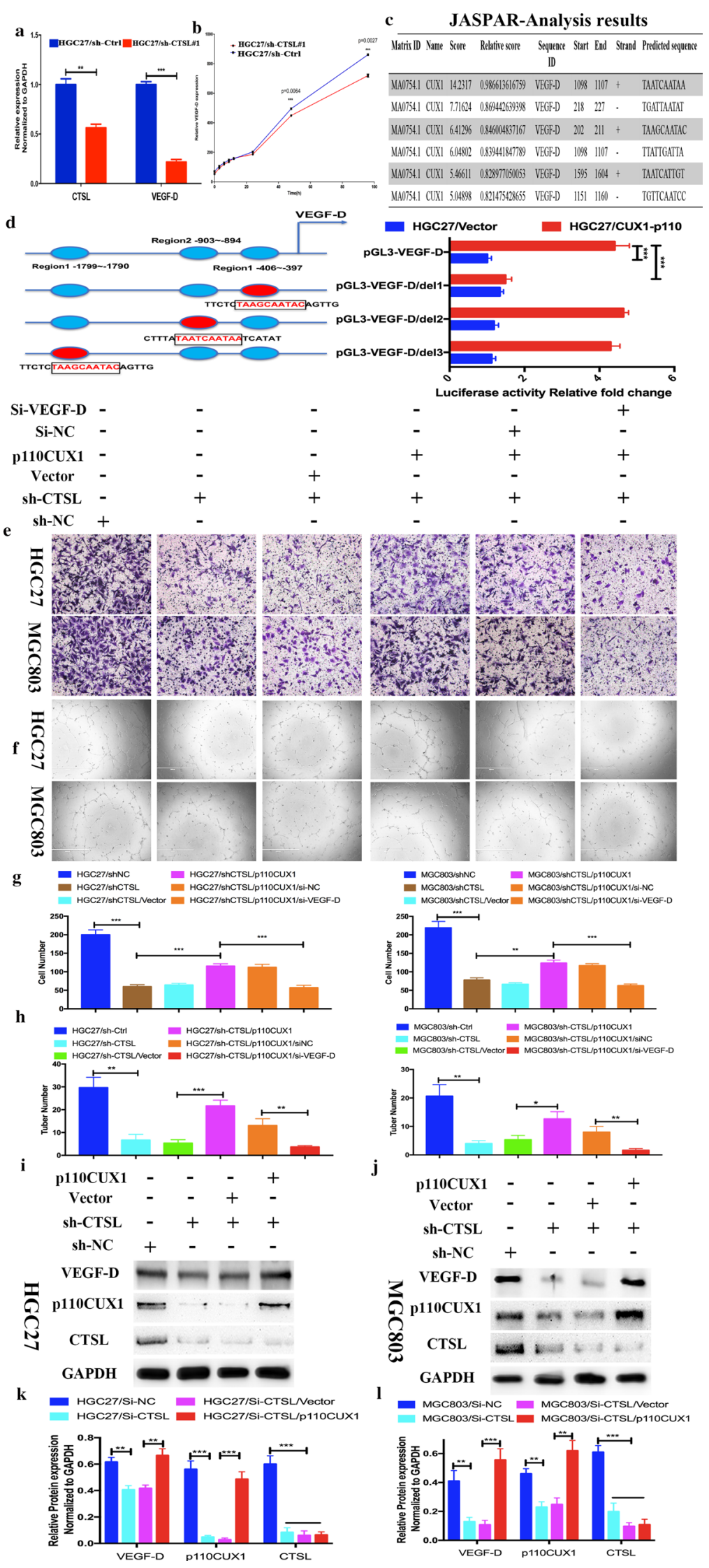


4Fig. 4 CTSL promoted the transcriptional activation of VEGF-D in GC cells by the processing of CUX1. a qRT-PCR assays showed that the mRNA levels of VEGF-D were obviously downregulated in the HGC27/sh-CTSL group compared with the HGC27/sh-Ctrl group. b ELISA demonstrated that the secretion of VEGF-D was decreased in the HGC27/sh-CTSL group compared with the HGC27/sh-Ctrl group. c The predicted potential binding sites between CDP/CUX and VEGF-D. d Deletion analysis at positions -406 to $-397 \mathrm{bp}$ (designated site 1), -903 to -894 bp (designated site 2), and -1799 to -1790 bp (designated site 3) identified three CDP/CUX1-responsive regions in the human VEGF-D promoter (left). pGL3-VEGFD, pGL3-VEGF-D-del 1 (deletion at positions-1799 to-1790), pGL3-VEGF-D-del 2 (deletion at positions-903 to-894 bp), pGL3-VEGF-D-del 3 (deletion at positions - 406 to -397 bp) constructs, along with the pRL-TK plasmid, were transfected into HGC27-p110CUX1 cells and parental HGC27 cells, and then the relative luciferase activity was measured (right). e, g In vitro Transwell migration assay to investigate the effect of CM from GC cells (MGC803 and HGC27) after they were transfected with sh-CTSL, p110CUX1-expressing vector, and si-VEGF-D on the metastatic abilities of HUVEC. Data are presented as the mean $\pm \mathrm{SD} ; n=3$ independent experiments. f, $\mathbf{h}$ In vitro tubular formation to investigate the effect of CM from GC cells (MGC803 and HGC27) after they were transfected with sh-CTSL, p110CUX1-expressing vector, and si-VEGF-D on HUVEC tubule formation. Data are presented as the mean $\pm \mathrm{SD} ; n=3$ independent experiments. $\mathbf{i}-\mathbf{l}$ The expression levels of CTSL, p110CUX1, and VEGF-D proteins in MGC803 and HGC27 cells transfected with p110CUX1-expressing vector or CTSL shRNA were analyzed by Western blot. Data are presented as the mean $\pm \mathrm{SD} ; n=3$ independent experiments

\section{Discussion}

CTSL belongs to the papain-like family of cysteine proteases and is essential in the terminal degradation of proteins [33]. CTSL localizes primarily to the lysosomes and is also a constituent of the nuclear protein fraction. Although previous studies have shown that CTSL is upregulated in GC and could be identified as a marker of enhanced invasiveness of GC $[10,15,30]$, the function of nuclear CTSL in GC angiogenesis has not been determined. In this study, we found that nuclear CTSL is significantly upregulated in GC cancer and predicts poor prognosis of GC patients. Nuclear CTSL significantly promotes the angiogenic potential of HUVECs cells in vitro and in vivo by promoting $\mathrm{CDP} / \mathrm{Cux}$ degradation to the $\mathrm{p} 110 \mathrm{Cux} 1$ isoform, thus increasing the transcription of VEGF-D. In this study, we first showed that CTSL plays a facilitative role in regulating tumor angiogenesis of GC and that it could serve as an independent prognostic indicator and therapeutic target for GC.

Functions of cysteine cathepsins depend on their subcellular localization. For example, cysteine cathepsins are involved in cell death and inflammation in the cytoplasm, and they also regulate cell cycle in the nucleus and exert degradative roles in the extracellular environment [18]. CTSL is a ubiquitously expressed cysteine protease that localizes primarily to the lysosome. The role of CTSL originally was assumed to be terminal degradation of proteins in the lysosomal compartment. CTSL expression and subcellular localization, however, do not appear to be uniform. Haugen et al. identified CTSL as a constituent of the nuclear protein fraction [12]. In this study, we found that nuclear CTSL promoted angiogenesis by enhancing the cleavage of CDP/Cux to $110 \mathrm{Cux} 1$ in GC. Proteolytic processing is an important post-translational mechanism that modulates downstream signaling. CDP/Cux can be proteolytically processed by CTSL, thereby generating the $110 \mathrm{Cux} 1$ isoform that contains three DNA-binding domains, CR2, CR3, and HD. CDP/Cux can bind only transiently to DNA, and the consequence of proteolytic processing is to activate the ability of $\mathrm{CDP} / \mathrm{Cux}$ to interact stably with DNA. The $\mathrm{p} 110 \mathrm{Cux} 1$ processed isoform binds stably to DNA, and therefore, it can function like a classical transcription factor [23]. Thus, we provided a novel insight into the proteolytic activity of nuclear CTSL in GC cells. The mechanism by which nuclear CTSL regulates the progression of GC requires further study.

VEGF-D is a secreted glycoprotein that promotes growth of blood vessels (angiogenesis) and lymphatic vessels (lymphangiogenesis). VEGF-D enhances tumor growth and metastatic spread, which correlates with poor patient outcome and resistance to anti-angiogenic drugs. VEGFD activates VEGF receptors (VEGFR-2 and VEGFR-3) on the endothelium to promote the growth and remodeling of blood or lymphatic vessels [28]. VEGF-D has been reported to be an independent prognostic marker, which aids in the identification of patients with poor prognosis after curative resection of gastric adenocarcinomas. Consistent with the previous study, we found that VEGF-D promoted the angiogenesis in GC. Although the molecular mechanisms that regulate expression of VEGF-D have been gradually interpreted, they have been not fully understood in GC. In this study, we identified that p110 Cux, an isoform of CDP/ Cux 1, was a transcription factor that specifically promoted the transcription of VEGF-D. Thus, our data uncovered a novel mechanism underlying VEGF-D regulation in GC.

\section{Conclusion}

Our findings demonstrated that nuclear CTSL was significantly upregulated in GC tissues and enhanced the cleavage of CDP/Cux to $\mathrm{p} 110 \mathrm{Cux} 1$ isoform by its proteolytic activity in GC cells. Moreover, we identified for the first time that p110 Cux 1 could bind to the promoter of VEGF-D and subsequently activated VEGF-D transcription, which led to GC angiogenesis. 

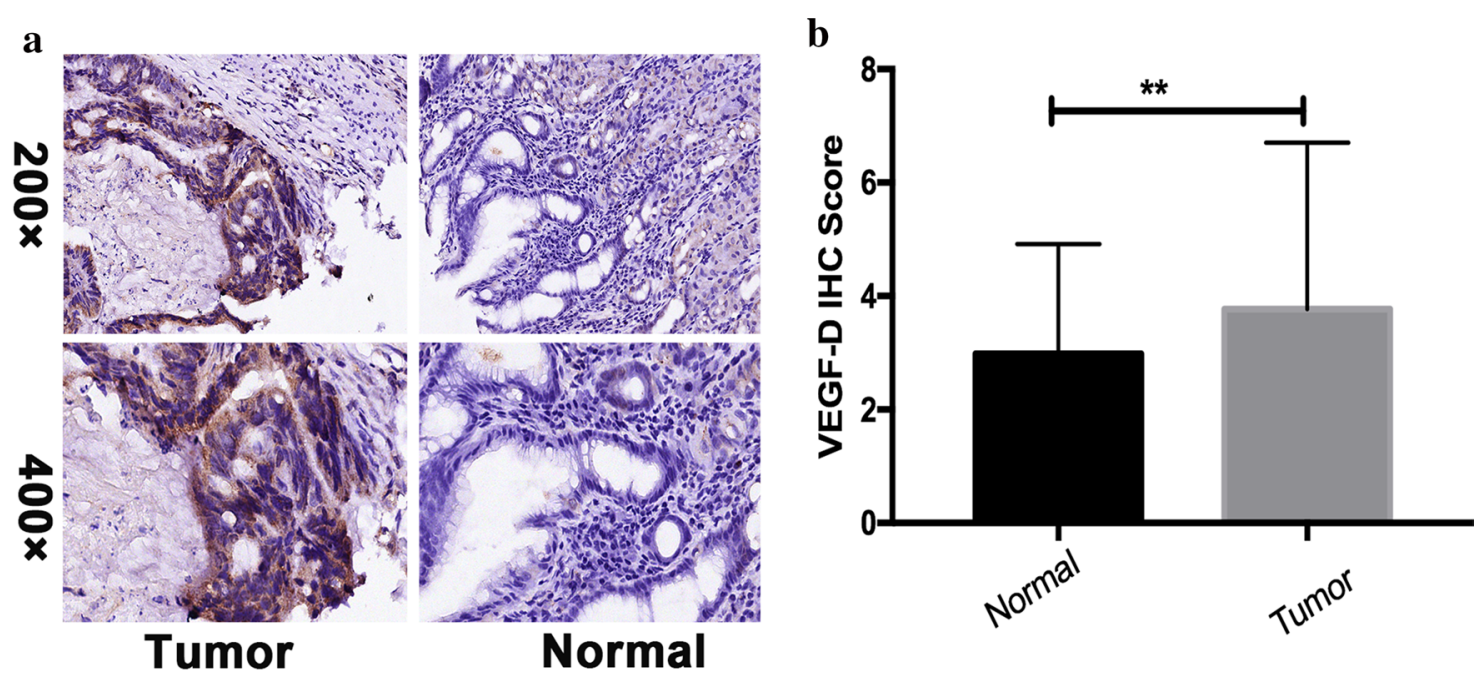

c

d
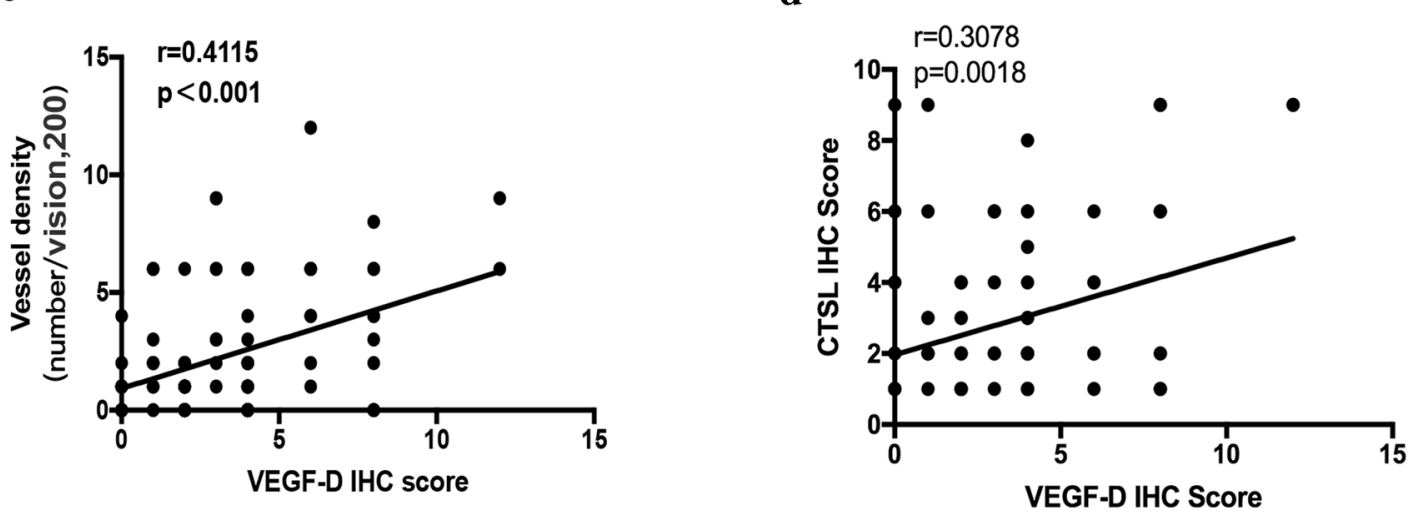

e

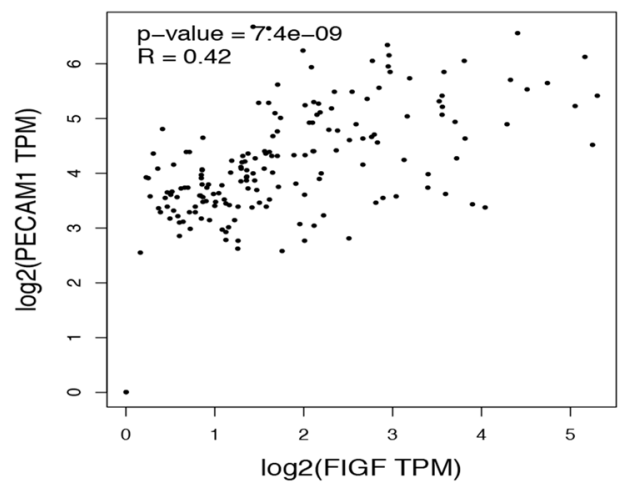

$\mathbf{f}$

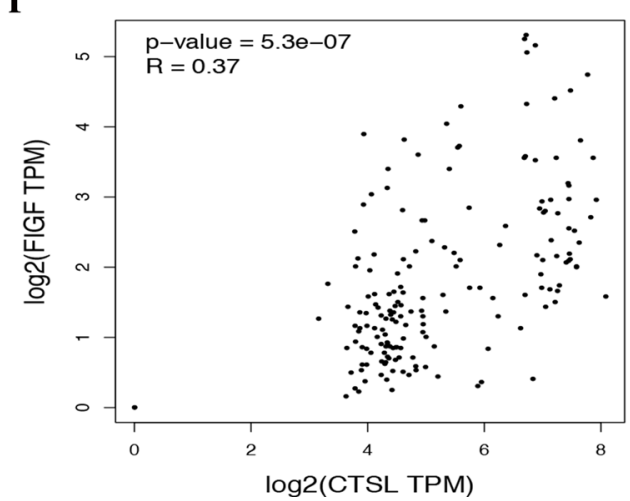

Fig. 5 CTSL was positively correlated with VEGF-D in GC tissues. a Representative IHC staining with VEGF-D antibody in GC and paired adjacent nontumor tissues. Magnification: $\times 200$ and $\times 400$. b Analysis of VD in 134 pairs of GC patients (GC cohort 1) showed that VEGF-D was significantly higher in GC tissues compared with adjacent nontumor tissues. c Correlation analysis of VEGF-D and

VD showed that VEGF-D was positively correlated with VD in GC. d Correlation analysis of VEGF-D and CTSL showed that VEGF-D was positively correlated with CTSL in GC. e Validation of the positive correlation between VEGF-D and VD in GEPIA. $\mathbf{f}$ Validation of the positive correlation between VEGF-D and CTSL in GEPIA 
Acknowledgements We thank Accdon (www.accdon.com) for its linguistic assistance during the preparation of this manuscript.

Author contributions LPS conceived the study, BYL carried out its design. TP, ZJY, ZJJ, XYW, and XFW performed the experiments. ZL, XYC, and QZ collected clinical samples. TP, ZJY, ZJJ, JFL, FYL, and ZYF analyzed the data and wrote the paper. LPS and BYL revised the paper. All authors read and approved the final manuscript.

Funding This work was supported by the National Natural Science Foundation of China (No. 81871902 and 81572798 to L. Su, No. 81672822 to Z. Zhu, No. 81772509 to B. Liu), Shanghai Municipal Education Commission-Gaofeng Clinical Medicine Grant Support (No. 20152505 to $\mathrm{L}$. Su).

Availability of data and materials All data and details can be obtained by contacting the corresponding author.

\section{Compliance with ethical standards}

Conflict of interest The authors declare no potential conflicts of interest.

Ethics approval and consent to participate All procedures performed in studies involving human participants were conducted in accordance with the Declaration of Helsinki and approved by the Human Ethics Committee of Ruijin Hospital (Shanghai JiaoTong University School of Medicine, Shanghai, China). All animal experiments were approved by the Animal Care and Use Committee of Ruijin Hospital.

Consent for publication All authors agree the publication of this study.

Open Access This article is licensed under a Creative Commons Attribution 4.0 International License, which permits use, sharing, adaptation, distribution and reproduction in any medium or format, as long as you give appropriate credit to the original author(s) and the source, provide a link to the Creative Commons licence, and indicate if changes were made. The images or other third party material in this article are included in the article's Creative Commons licence, unless indicated otherwise in a credit line to the material. If material is not included in the article's Creative Commons licence and your intended use is not permitted by statutory regulation or exceeds the permitted use, you will need to obtain permission directly from the copyright holder. To view a copy of this licence, visit http://creativecommons.org/licenses/by/4.0/.

\section{References}

1. Achen MG, Jeltsch M, Kukk E, Makinen T, Vitali A, Wilks AF, et al. Vascular endothelial growth factor D (VEGF-D) is a ligand for the tyrosine kinases VEGF receptor 2 (Flk1) and VEGF receptor 3 (Flt4). Proc Natl Acad Sci USA. 1998;95:548-53.

2. Bernards N, Creemers GJ, Nieuwenhuijzen GA, Bosscha K, Pruijt JF, Lemmens VE. No improvement in median survival for patients with metastatic gastric cancer despite increased use of chemotherapy. Ann Oncol. 2013;24:3056-60.

3. Bray F, Ferlay J, Soerjomataram I, Siegel RL, Torre LA, Jemal A. Global cancer statistics 2018: GLOBOCAN estimates of incidence and mortality worldwide for 36 cancers in 185 countries. CA Cancer J Clin. 2018;68:394-424.
4. Brix K, Dunkhorst A, Mayer K, Jordans S. Cysteine cathepsins: cellular roadmap to different functions. Biochimie. 2008;90:194-207.

5. Chapman HA, Riese RJ, Shi GP. Emerging roles for cysteine proteases in human biology. Annu Rev Physiol. 1997;59:63-88.

6. Chen S, Dong H, Yang S, Guo H. Cathepsins in digestive cancers. Oncotarget. 2017;8:41690-700.

7. Chen W, Zheng R, Baade PD, Zhang S, Zeng H, Bray F, et al. Cancer statistics in China, 2015. CA Cancer J Clin. 2016;66:115-32.

8. Cheng X, Jin Z, Ji X, Shen X, Feng H, Morgenlander W, et al. ETS variant 5 promotes colorectal cancer angiogenesis by targeting platelet-derived growth factor BB. Int J Cancer. 2019;145:179-91.

9. Denhardt DT, Hamilton RT, Parfett CL, Edwards DR, St Pierre $\mathrm{R}$, Waterhouse $\mathrm{P}$, et al. Close relationship of the major excreted protein of transformed murine fibroblasts to thiol-dependent cathepsins. Cancer Res. 1986;46:4590-3.

10. Dohchin A, Suzuki JI, Seki H, Masutani M, Shiroto H, Kawakami Y. Immunostained cathepsins B and L correlate with depth of invasion and different metastatic pathways in early stage gastric carcinoma. Cancer. 2000;89:482-7.

11. Fei X, Qin Z, Liang Z. Contribution of CDP/Cux, a transcription factor, to cell cycle progression. Acta Biochim Biophys Sin (Shanghai). 2007;39:923-30.

12. Haugen MH, Johansen HT, Pettersen SJ, Solberg R, Brix K, Flatmark K, Maelandsmo GM (2013) Nuclear legumain activity in colorectal cancer. PloS one 8(1):e52980. https://doi. org/10.1371/journal.pone.005298

13. He B, Xiao YF, Tang B, Wu YY, Hu CJ, Xie R, et al. hTERT mediates gastric cancer metastasis partially through the indirect targeting of ITGB1 by microRNA-29a. Sci Rep. 2016;6:21955.

14. Ikeguchi M, Fukuda K, Oka S, Hisamitsu K, Katano K, Tsujitani S, et al. Micro-lymph node metastasis and its correlation with cathepsin D expression in early gastric cancer. J Surg Oncol. 2001;77:188-94

15. Kielan W, Suzanowicz J, SiewiNski M, Saleh Y, Janocha A, Skalski A, et al. Evaluation of changes in the activity of proteolytic enzymes and their inhibitors in the processes that accompany the growth of gastric cancer. Gastric Cancer. 2004;7:17-23.

16. Kim KJ, Li B, Winer J, Armanini M, Gillett N, Phillips HS, et al. Inhibition of vascular endothelial growth factor-induced angiogenesis suppresses tumour growth in vivo. Nature. 1993;362:841-4.

17. Kopfstein L, Veikkola T, Djonov VG, Baeriswyl V, Schomber T, Strittmatter K, et al. Distinct roles of vascular endothelial growth factor-D in lymphangiogenesis and metastasis. Am J Pathol. 2007; 170:1348-61.

18. Kramer L, Turk D, Turk B. The future of cysteine cathepsins in disease management. Trends Pharmacol Sci. 2017;38:873-98.

19. Liu WL, Liu D, Cheng K, Liu YJ, Xing S, Chi PD, et al. Evaluating the diagnostic and prognostic value of circulating cathepsin $\mathrm{S}$ in gastric cancer. Oncotarget. 2016;7:28124-38.

20. Lu HJ, Yan J, Jin PY, Zheng GH, Qin SM, Wu DM, et al. MicroRNA-152 inhibits tumor cell growth while inducing apoptosis via the transcriptional repression of cathepsin $\mathrm{L}$ in gastrointestinal stromal tumor. Cancer Biomark. 2018;21:711-22.

21. Melincovici CS, Bosca AB, Susman S, Marginean M, Mihu C, Istrate $\mathrm{M}$, et al. Vascular endothelial growth factor (VEGF) - key factor in normal and pathological angiogenesis. Rom J Morphol Embryol. 2018;59:455-67.

22. Miyamoto K, Iwadate M, Yanagisawa Y, Ito E, Imai J, Yamamoto $\mathrm{M}$, et al. Cathepsin $\mathrm{L}$ is highly expressed in gastrointestinal stromal tumors. Int J Oncol. 2011;39:1109-15.

23. Moon NS, Berube G, Nepveu A. CCAAT displacement activity involves CUT repeats 1 and 2, not the CUT homeodomain. J Biol Chem. 2000;275:31325-34. 
24. Patel S, Homaei A, El-Seedi HR, Akhtar N. Cathepsins: Proteases that are vital for survival but can also be fatal. Biomed Pharmacother. 2018;105:526-32.

25. Pislar A, Jewett A, Kos J. Cysteine cathepsins: their biological and molecular significance in cancer stem cells. Semin Cancer Biol. 2018;53:168-77.

26. Ramdzan ZM, Nepveu A. CUX1, a haploinsufficient tumour suppressor gene overexpressed in advanced cancers. Nat Rev Cancer. 2014;14:673-82.

27. Shibuya M. Vascular endothelial growth factor and its receptor system: physiological functions in angiogenesis and pathological roles in various diseases. J Biochem. 2013;153:13-9.

28. Stacker SA, Caesar C, Baldwin ME, Thornton GE, Williams RA, Prevo R, et al. VEGF-D promotes the metastatic spread of tumor cells via the lymphatics. Nat Med. 2001;7:186-91.

29. Stratigopoulos G, LeDuc CA, Cremona ML, Chung WK, Leibel RL. Cut-like homeobox 1 (CUX1) regulates expression of the fat mass and obesity-associated and retinitis pigmentosa GTPase regulator-interacting protein-1-like (RPGRIP1L) genes and coordinates leptin receptor signaling. J Biol Chem. 2011;286:2155-70.

30. Sudhan DR, Siemann DW. Cathepsin L targeting in cancer treatment. Pharmacol Ther. 2015;155:105-16.

31. Turk V, Turk B, Turk D. Lysosomal cysteine proteases: facts and opportunities. EMBO J. 2001;20:4629-33.
32. Uloza V, Kuzminiene A, Palubinskiene J, Balnyte I, Uloziene I, Valanciute A. Model of human recurrent respiratory papilloma on chicken embryo chorioallantoic membrane for tumor angiogenesis research. Histol Histopathol. 2017;32:699-710.

33. Vizovisek M, Fonovic M, Turk B. Cysteine cathepsins in extracellular matrix remodeling: extracellular matrix degradation and beyond. Matrix Biol. 2019;75-76:141-59.

34. Von Marschall Z, Scholz A, Stacker SA, Achen MG, Jackson DG, Alves F, et al. Vascular endothelial growth factor-D induces lymphangiogenesis and lymphatic metastasis in models of ductal pancreatic cancer. Int J Oncol. 2005;27:669-79.

35. Wagner AD, Syn NL, Moehler M, Grothe W, Yong WP, Tai BC, et al. Chemotherapy for advanced gastric cancer. Cochrane Database Syst Rev. 2017;8:CD004064.

36. Yu Z, Li Z, Wang C, Pan T, Chang X, Wang X, et al. Oncostatin $M$ receptor, positively regulated by SP1, promotes gastric cancer growth and metastasis upon treatment with Oncostatin M. Gastric Cancer. 2019;22:955-66.

Publisher's Note Springer Nature remains neutral with regard to jurisdictional claims in published maps and institutional affiliations. 